Article

\title{
Construction and Simulation of Composite Measures and Condensation Model for Designing Probabilistic Computational Applications
}

\author{
Susmit Bagchi \\ Department of Aerospace and Software Engineering (Informatics), Gyeongsang National University, \\ Jinju 660701, Korea; profsbagchi@gmail.com
}

Received: 13 October 2018; Accepted: 13 November 2018; Published: 15 November 2018

\begin{abstract}
The probabilistic algorithms are widely applied in designing computational applications such as distributed systems and probabilistic databases, to determine distributed consensus in the presence of random failures of nodes or networks. In distributed computing, symmetry breaking is performed by employing probabilistic algorithms. In general, probabilistic symmetry breaking without any bias is preferred. Thus, the designing of randomized and probabilistic algorithms requires modeling of associated probability spaces to generate control-inputs. It is required that discrete measures in such spaces are computable and tractable in nature. This paper proposes the construction of composite discrete measures in real as well as complex metric spaces. The measures are constructed on different varieties of continuous smooth curves having distinctive non-linear profiles. The compositions of discrete measures consider arbitrary functions within metric spaces. The measures are constructed on 1-D interval and 2-D surfaces and, the corresponding probability metric product is defined. The associated sigma algebraic properties are formulated. The condensation measure of the uniform contraction map is constructed as axioms. The computational evaluations of the proposed composite set of measures are presented.
\end{abstract}

Keywords: measure spaces; probability; metric spaces; randomized algorithms; monotone

\section{Introduction}

The applications of probabilistic models in designing algorithms and solving computational problems are pervasive in nature. The applications of randomized or probabilistic algorithms are found in distributed systems for determining consensus and agreement in the presence of random failures of nodes as well as networks [1]. The probabilistic databases require specific and computable models to measure uncertainties in noisy datasets [2,3]. The probabilistic algorithms are designed for events segregation in a computing system and to perform cluster analysis in probabilistic databases [4]. Furthermore, the computation of symmetry breaking in distributed systems requires probabilistic algorithms [5]. In general, the deterministic algorithms offer relatively optimized solutions, having higher computational cost. Often, the deterministic algorithms employ restrictive approximate models of the problems leading to over simplification [6]. However, the probabilistic algorithms are more suitable to formulate algorithmic models of systems having inherent uncertainties [7]. For example, the algorithmic measurements of the occurrence of certain types or classes of events in a set of random events employ probabilistic models [8]. The algorithm randomly assigns a set of initial values to the elements of a set of events prior to computing the maximal independent set. In general, the probabilistic and randomized algorithms offer acceptable solutions at reduced computational cost in comparison to deterministic algorithms $[5,9,10]$. In the extreme point identification algorithm within $d$-dimensional real space for cluster analysis, the probabilistic estimation models are employed [9]. The other 
examples of probabilistic distributed algorithms are probabilistic graph coloring [11] and computation of symmetry breaking in distributed computing systems [12]. The model checking algorithms are generally designed using temporal logic. However, the probabilistic models involving discrete-time Markov chains can be employed to realize probabilistic model checking as well as verification [13]. The designing of probabilistic computing systems requires the formulation of appropriate models of probability measures [14]. The properties of the models vary depending upon the associated measure spaces. Researchers have proposed computable metric spaces with probabilistic measures as well as associated binary representations for applications in randomized algorithms [15]. Thus, one can view the metric spaces as computable entities with appropriate representations. Moreover, the models of discrete measures are important for designing and analyzing probabilistic computing systems as well as distributed algorithms.

\section{Motivation}

The probabilistic algorithms are effective solutions to design computational applications under uncertainties offering acceptable results [16-18]. The randomized and probabilistic algorithms require input data and a random bit-stream or set of keys to compute on datasets [10]. The outputs of randomized algorithms vary depending on the random input values and the key, which changes the execution trajectory of the respective algorithm. The generations of a set of random keys having specific characteristics require corresponding probability measure spaces. The common design patterns of probabilistic and randomized algorithms involve the formulation of a probability model to generate and assign values to a set of events having certain significance depending upon the applications. The correctness analysis of randomized algorithms requires involvement of probability spaces of computation [5]. The probability measure spaces have applications in modeling and designing probabilistic systems such as probabilistic distributed algorithms [1,19]. It is often desired that discrete measures associated with probability follow some computable smooth function while sampling random values in order to reduce computational cost and to enhance easy realizations of software systems. This paper proposes a set of new models to construct composite discrete measures on arbitrary smooth functions. The proposed model considers real and complex $(R, C)$ metric spaces. The corresponding analytical properties of the model are formulated using sigma algebraic constructs. The monotone space properties are presented. The condensation measure of the uniform contraction map is formulated. The main contributions of the paper are as follows.

- A generalized computational model of composite discrete measures on arbitrary smooth functions is formulated in real and complex metric spaces.

- It is illustrated that the model can operate on linear, non-linear and arbitrary smooth functions.

- The operational modes and properties of the measures on z-plane are constructed.

- The construction of composite measures on a real 2-D surface is proposed.

- The concept of condensation measure of uniform contraction map and the associated properties are presented.

The rest of the paper is organized as follows. Section 2 presents related work. Section 3 presents a formulation of the model and its properties. Section 4 introduces monotone class and metric product forms. The formulation of a composite measure on 2-D real surface and condensation measure of the contraction map are presented in Section 5. The computational evaluations of the models are illustrated in Section 6. Section 7 depicts comparative analysis. Finally, Section 8 concludes the paper.

\section{Related Work}

The models of various probability measures are applied in designing distributed algorithms and systems. The formal modeling and analysis of distributed detection of a set of intersections are performed based on a probabilistic model [20]. The main aim of applications of probabilistic models is to reduce computational complexity exhausting the entire search space, while achieving acceptable 
results. The applications of probability measures are found in designing election algorithms in complex networks. For example, a probabilistic distributed algorithm is designed for uniform election in a polyo-triangular graph [21]. The construction of discrete probability models requires associated discrete measures as a foundation [22]. Interestingly, the discrete measures can be formulated in metric spaces having specialized properties. The metric space is a space on a metrizable set equipped with axioms. A special class of metric spaces is called complete separable spaces structured with weak topology [23]. In general, the mapping on such metric spaces is a continuous and onto function. However, the continuous bilinear onto mapping in a space is not necessarily to be open in $(0,0)$. The concept of formulating probability measures over the metric spaces is explained in Reference [24].

The construction of probability measures over a set of convex bodies in metric spaces is proposed by researchers [25]. The model considers $n$-dimensional Euclidean spaces and the probability space of norms are defined by unit ball. The formulation covers smooth bodies and the neighborhood with positive probability measures. The research on algorithmic randomness in Cantor space is conceptualized incorporating the random infinite sequences. It is noted that the concepts of game-theoretic as well as measure-theoretic algorithmic randomness converge to a common space [26]. Often, the metric spaces and associated probability measures are computable in nature [27]. The computability of Borel probability measured over the generalized compact spaces is investigated [28]. The computable probability measures covering metric spaces are proposed, having applications in algorithmic randomness [15]. In this case, the concept of binary representation is introduced in Cantor space, which allows the identification of computable probability spaces. This model helps in applying the algorithmic randomness to a computable probability space.

In general, the algorithmic randomness is tractable and computable when the covering space is comprised of a set of finite or infinite sequences having a uniform probability distribution (or any other computable distributions). The method for uniform testing of algorithmic randomness covering any general metric spaces is analyzed [29]. The test considers the recognizable Boolean inclusion principles. However, the universal randomness testing under weak conditions is presented in the literature [27]. The cellular automata-based algorithmic randomness configurations are proposed including randomness tests [30]. The results illustrate that surjective maps in cellular automata can be a basis for generating randomness in full shift spaces.

\section{Formulation of Model}

In this section, sets of models are formulated for computing the composite discrete measures on functions over the metric spaces having certain properties. The model considers two metric spaces such as a function metric space on 1-D real $(R)$ and a complex metric space in z-plane $(C)$. The measure on 1-D real is further extended to 2-D real metric surface equipped with suitable continuous functions.

\subsection{Composite Measures in Real Metric Space}

Initially, it is considered that underlying metric space is a 1-D real space maintaining standard metrizability conditions. Let $f(x \in R) \in R$ be a smooth continuous function in the region $D_{a}, D_{b} \in R$ and, $x_{j} \in\left[D_{a}, D_{b}\right]$ where, $D_{b}>D_{a}$ and $j \in N$. Let $(S, d)$ be a function metric space on 1-D real with $S=\left\{e_{j}: e_{j}=f(x) \delta\left(x-x_{j}\right), e_{j} \in R\right\}$ such that, $\int_{-\infty}^{+\infty} \delta(x) d x=1$ and, $\forall e_{j}, e_{k} \in S: d\left(e_{j}, e_{k}\right)=\left|e_{j}-e_{k}\right|$. The set $A \subset R$ and a binary relation $\Re_{B} \subset A \times S$ are formulated having conditions as mentioned below,

$$
\begin{aligned}
& \forall y_{j} \in A, \exists\left(y_{j}, e_{j}\right) \in \Re_{B}: \\
& \left(y_{j} \notin R^{+} \cup\{0\}\right) \Rightarrow\left(f\left(x_{j}\right) \leq 0\right) \\
& \text { and, } \\
& \left(y_{j} \in R^{+}\right) \Rightarrow\left(f\left(x_{j}\right)>0\right)
\end{aligned}
$$


The discrete measure over $(S, d)$ covering $A$ is defined as,

$$
p: \Re_{B} \rightarrow[0,1]
$$

The covering measure is closed and bounded. However, the measure is generalized in nature and the conditions of probabilistic measures are not imposed on it.

\subsection{Composite Measures in Complex Metric Space}

In this case, the metric space is considered to be a complex 2-D plane having a definable and computable norm. Let $\left(X_{c}, d_{c}\right)$ be a complex metric space on z-plane where $X_{c} \subset C$ and $d_{c}\left(z_{j}, z_{k}\right)=|| z_{j}|-| z_{k}||,\left\{z_{j}, z_{k}\right\} \subset X_{c}$. A function $f_{c}: X_{c} \rightarrow R$ is defined considering respective inner-product as,

$$
\forall z_{j} \in X_{c}: f_{c}\left(z_{j}\right)=z_{j} z_{j} *
$$

By denoting $S_{f}=\left\{f_{c}\left(z_{j}\right): z_{j} \in X_{c}\right\}$, the discrete measure on the complex metric space covering $A \subset R$ can be formulated as,

$$
p_{c}: A \times S_{f} \rightarrow[0,1]
$$

In this case, the covering measure is confined within a unit interval without any specific restriction on measurability.

\subsection{Properties of Composite Measure in $\sigma-$ Algebra}

The discrete measures can be evaluated at uncorrelated discrete sample points. Otherwise, it can be evaluated cumulatively under correlation. Suppose, $A \subset R$ is a discrete countable finite set and $g: A \rightarrow S$ be an injective function such that the following biconditional is maintained,

$$
\left[r \in \Re_{B}\right] \Leftrightarrow[r \in A \times g(A)]
$$

Hence, $B_{\sigma}=P(A)$ is a discrete $\sigma-$ algebra on $A$. As a result, three different conditions on $B_{\sigma}$ can be formulated related to corresponding probability measures. A $B_{\sigma}$ is locally complete with respect to $p($.$) iff the following condition is maintained,$

$$
\forall H \in B_{\sigma}: \sum_{\forall y \in H} p(y, g(y))=1
$$

However, the distributive completeness of the discrete measure can be determined by using sampling from the members of $B_{\sigma}$. Suppose, $K \subset B_{\sigma}$ and $A_{\sigma}=\left\{y_{j} \in A\right\}$ such that the following property is maintained,

$$
\begin{aligned}
& \forall y_{j}, y_{k} \in A_{\sigma}: \exists H_{j}, H_{k} \in K: \\
& \left(y_{j} \in H_{j}\right) \wedge\left(y_{k} \in H_{k}\right) \Leftrightarrow\left(y_{j}, y_{k} \notin H_{j} \cap H_{k}\right)
\end{aligned}
$$

Thus, a $B_{\sigma}$ is defined as distributive complete with respect to the associated probability measure $p($.$) iff the following condition is maintained,$

$$
\exists A_{\sigma} \subset A: \sum_{\forall y \in A_{\sigma}} p(y, g(y))=1
$$

Furthermore, the completeness in 1-D real under correlated cumulative measurement can be determined by maintaining $E_{j} \in \Re_{B}: \sum_{\forall y_{j} \in A} p\left(E_{j}\right)=1$. 
The $\sigma$ - algebra for $p_{c}($.$) in complex metric space can be determined with the respective injective$ function $g_{c}: A \rightarrow S_{f}$. Let $\Re_{H} \subset A \times S_{f}$ such that $\forall r_{j} \in \Re_{H}: r_{j}=\left(y_{j}, g_{c}\left(y_{j}\right)\right)$ where $y_{j} \in H \in B_{\sigma}$. Thus, $B_{\sigma}$ is locally complete in z-plane with respect to $p_{c}($.$) iff the following condition is maintained,$

$$
\forall H \in B_{\sigma}: \sum_{\forall y_{j} \in H} p_{c}\left(r_{j} \in \Re_{H}\right)=1
$$

Considering $K \subset B_{\sigma}$ for the probability measure in z-plane it can be derived as $\exists H_{j}, H_{k} \in K$ such that $A_{\sigma}=\left\{y_{j} \in A\right\}$ satisfying Equation (7). Suppose, $\overline{\Re_{H}} \subset A_{\sigma} \times g_{c}\left(A_{\sigma}\right)$ and $\forall r_{j} \in \overline{\Re_{H}}: r_{j}=$ $\left(y_{j} \in A_{\sigma}, g_{c}\left(y_{j}\right)\right)$. A $B_{\sigma}$ is considered to be distributive complete in z-plane with respect to $p_{c}($.$) iff the$ following property is maintained,

$$
\exists A_{\sigma} \subset A: \sum_{\forall y_{j} \in A_{\sigma}} p_{c}\left(r_{j} \in \overline{\Re_{H}}\right)=1
$$

Furthermore, if $\Re_{A} \subset A \times S_{f}$ such that $\forall r_{j} \in \Re_{A}: r_{j}=\left(y_{j} \in A, g_{c}\left(y_{j}\right)\right)$ then $B_{\sigma}$ is complete under correlation with respect to probability measure $p_{c}($.$) in complex metric space iff the following$ condition is satisfied,

$$
\forall r_{j} \in \Re_{A}: \sum_{\forall y_{j} \in A} p_{c}\left(r_{j} \in \Re_{A}\right)=1
$$

\section{Computing Probability-Metric Product}

This section enumerates the monotone classes formed by the composite discrete measures and formulates the concept of the probability-metric product. Suppose $B_{\sigma}$ is locally complete with respect to probability measures $p($.$) or p_{c}($.$) in 1-D real or complex space, respectively. Let the local set of$ discrete measures be defined as given below, considering $p_{\sigma}($.$) denoting either p($.$) or p_{c}($.$) depending$ on respective metric spaces,

$$
T_{j}=\left\{p_{\sigma}\left(r_{j}\right): H_{j} \in B_{\sigma}\right\}
$$

The set $M_{j} \subseteq P\left(T_{j}\right)$ is a monotone class of $T_{j}$ for the corresponding composite probability measure in respective metric spaces. It can be easily verified that,

$$
\bigcup_{j=1}^{\left|B_{\sigma}\right|} M_{j} \subseteq P\left(\bigcup_{j=1}^{\left|B_{\sigma}\right|} T_{j}\right)
$$

The metric ratio is a ratio between distance metric and sample points in a metric space. The metric ratio $\chi$ is defined as given below considering $d_{\sigma}\left(a_{j}, a_{k}\right)$ denoting either $d($.$) in 1-D real metric space$ with $\left\{a_{j}, a_{k}\right\} \subset S$ or $d_{c}($.$) in z-plane with \left\{a_{j}, a_{k}\right\} \subset X_{c}$,

$$
\forall y_{j}, y_{k} \in A: \chi=\frac{d_{\sigma}\left(a_{j}, a_{k}\right)}{y_{j}-y_{k}}
$$

Thus, the probability-metric product $\left(\lambda_{S}\right.$ in case of 1-D real space and, $\lambda_{C}$ in case of complex space) can be computed in real and complex metric spaces as,

$$
\begin{aligned}
& \forall a_{j}, a_{k} \in S: \exists y_{j}, y_{k} \in A: \\
& \lambda_{S}=\chi\left(p\left(y_{j}, g\left(y_{j}\right)\right)-p\left(y_{k}, g\left(y_{k}\right)\right)\right) \\
& \text { and }, \\
& \forall a_{j}, a_{k} \in X_{c}: \exists y_{j}, y_{k} \in A: \\
& \lambda_{C}=\chi\left(p_{c}\left(y_{j}, g_{c}\left(y_{j}\right)\right)-p_{c}\left(y_{k}, g_{c}\left(y_{k}\right)\right)\right)
\end{aligned}
$$

The product determines dynamics of variations of distributions on a curve or on a surface, given a measure space. 


\section{Discrete Measure on 2-D Real Surface}

A 2-D extension of Dirac function can be given as $\delta(x, y)$ such that $\int_{-\infty}^{+\infty} \int_{-\infty}^{+\infty} \delta(x, y) d x d y=1$. Let $I \subset R$ and $J \subset R$ be two intervals and the metric space in 2-D is defined over $I \times J \subset R^{2}$. Furthermore, a real valued function $f: R^{2} \rightarrow R$ is given as, $\forall \beta \in I \times J: f(\beta) \in R$, which is computable in corresponding metric space. Let a set of samples derived from the surface of the measurement be given by,

$$
X_{2 D}=\{f(\beta) \delta(x, y): x \in I \wedge y \in J\}
$$

Thus, given a real valued continuous function $g:(A \subset R) \rightarrow R$, the discrete measure of $g($.$) on$ the surface of $f($.$) can be computed as,$

$$
\begin{aligned}
& B_{X} \subseteq\left\{X_{2 D} \times g(A)\right\}, \\
& p_{2 D}: B_{X} \rightarrow[0,1]
\end{aligned}
$$

This indicates that it is possible to discretely measure a function on a 2-D real metric space if the surface of measurement and domains are known a priori.

However, if $f: R^{2} \rightarrow R$ is separable then the discrete measure can be computed in product form. Let $u: R \rightarrow R$ and $v: R \rightarrow R$ be two arbitrary continuous real valued functions. If $f($.$) is separable$ such that $\forall \beta \in I \times J, \exists x \in I, \exists y \in J: f(\beta)=u(x) v(y)$ then the separable set of samples from the measure surface can be given as,

$$
\begin{aligned}
& X_{2 D x}=\{u(x) \delta(x): x \in I\}, \\
& X_{2 D y}=\left\{v(y) \delta(y): y \in J \wedge \int_{-\infty}^{+\infty} \delta(y) d y=1\right\}, \\
& X_{x y}=\{u(x) v(y): f(\beta)=u(x) v(y) \wedge \beta=(x, y)\}
\end{aligned}
$$

Furthermore, the composite measure of $g($.$) can be formulated on the discrete points on measure$ surface as,

$$
\begin{aligned}
& B_{x y} \subseteq\left\{X_{x y} \times g(A)\right\}, \\
& p_{x y}: B_{x y} \rightarrow[0,1]
\end{aligned}
$$

Thus, the construction of discrete measure on the surface can be performed depending on the characteristics of functions.

\section{Contraction and Condensation Measure}

Let $\left(X, d_{x}\right)$ be a metric space and $A \subset X$ be compact. Let an identity map be defined as, $i_{x}: X \rightarrow X$. A uniform contraction map is given as, $\theta_{c}: A \rightarrow(B \subset X)$, such that following axiom is satisfied, $\forall x, y \in A, d_{x}(x, y) \geq d_{x}\left(\theta_{\mathcal{c}}(x), \theta_{\mathcal{c}}(y)\right)$.

The uniform contraction is a condensation if the following axioms are maintained, where $B \subset A$,

$$
\begin{aligned}
& n_{i}, k \in Z^{+}, \\
& \exists n_{i} \in(1,+\infty), \forall k \leq n_{i}: \theta_{c}^{k}(A) \subset B, \\
& \forall k>n_{i}, \theta_{c}^{k}(A) \subseteq i_{x}(C \subset B)
\end{aligned}
$$

A compact set $D_{n} \subset A$ is called as $n$-condensation complete if, $D_{n} \subseteq \bigcap_{j=1}^{n} \theta_{c}^{j}(A)$. 
Thus, the finite condensation measure is defined as $\mu_{c}: X \rightarrow[0,+\infty)$ having the following properties,

$$
\begin{aligned}
& \forall k, j \in Z^{+}: \mu_{c}(A)>\mu_{c}\left(\theta_{c}^{k}(A)\right), \\
& \forall k>n_{i}, j>1: \mu_{c}\left(\theta_{c}^{k}(A)\right)=\mu_{c}\left(\theta_{c}^{k+j}(A)\right), \\
& \mu_{c}(A)=\mu_{c}\left(\bigcup_{i=0}^{+\infty} \theta_{c}^{i}(A)\right), \\
& \mu_{c}(A)<\sum_{i=0}^{+\infty} \mu_{c}\left(\theta_{c}^{i}(A)\right), \\
& \mu_{c}\left(\theta_{c}^{k}(A)\right)<\sum_{j=1}^{+\infty} \mu_{c}\left(\theta_{c}^{k+j}(A)\right)
\end{aligned}
$$

Furthermore, it can be derived that, $\mu_{c}\left(D_{n}\right)>\mu_{c}\left(D_{m}\right)$, where $k>m>n$.

\section{Computational Evaluation}

The composite discrete measures are evaluated by using computational methods considering various functions having different properties. The profile of distribution of numerically mapped values of keys of set $A$ in 1-D real is illustrated in Figure 1 spanning negative and positive domains.

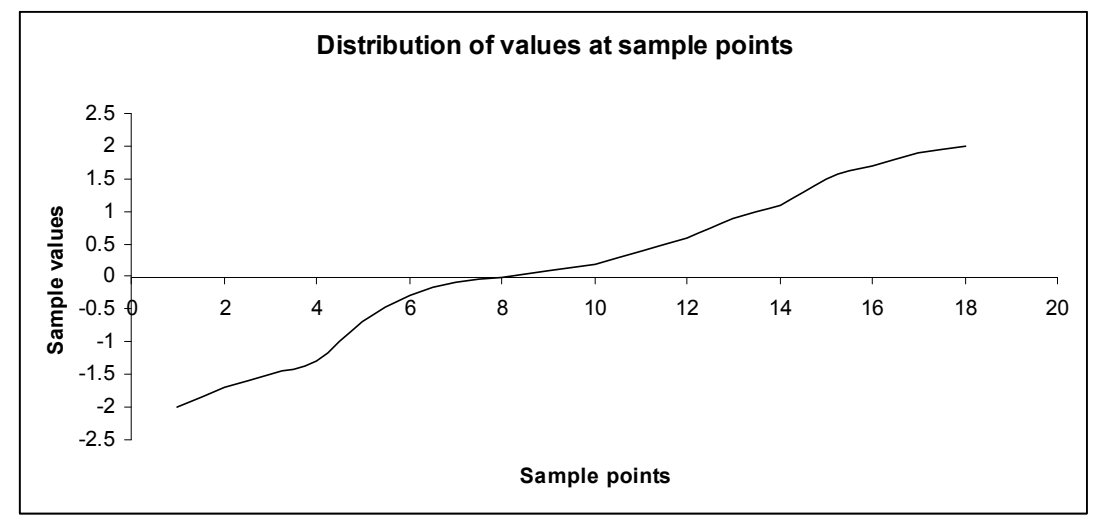

Figure 1. The distribution of $A$ for discrete measure.

The computational evaluations of discrete measures are carried out on non-linear smooth curves, linear smooth curves, periodic functions and on the function of complex numbers. The software implementation of computational evaluation is realized in $C$ language using installed standard math-libraries to generate numerical data sets. The implementation in $\mathrm{C}$ has offered greater flexibility in software design to carry out numerical analysis by generating data sets. However, the numerical analysis is not particularly dependent on programming languages and the proposed computational models can also be realized in any other suitable software platforms of choice.

\subsection{Evaluation on Linear Smooth Curve}

The computational evaluations are carried out considering linear smooth curves having two different slopes. In the first case, the slope is slant and in the second case, the slope is steep. The profile of the function $f(x)=a x$ is illustrated in Figure 2 considering $a=2$ and $a=3$.

The composite discrete measure on the curve is defined as given below,

$$
p\left(y_{j}, e_{j}\right)=\left\{\begin{array}{l}
\left|y_{j} /\left(y_{j}+e_{j}-1\right)\right|, y_{j}, e_{j} \leq 0 \\
\left|y_{j} /\left(y_{j}+e_{j}+1\right)\right|, y_{j}, e_{j}>0
\end{array}\right.
$$






Figure 2. Linear smooth curves with slant and steep slopes.

The variations of discrete measures on the slant linear curve and the steep linear curve are illustrated in Figures 3 and 4, respectively.

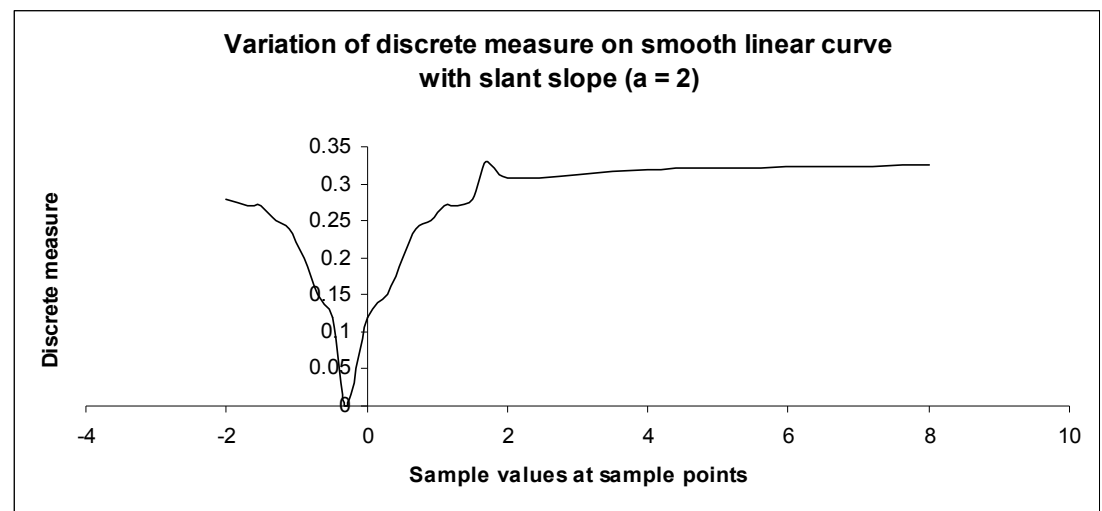

Figure 3. Variation of discrete measure on linear curve with slope $a=2$.

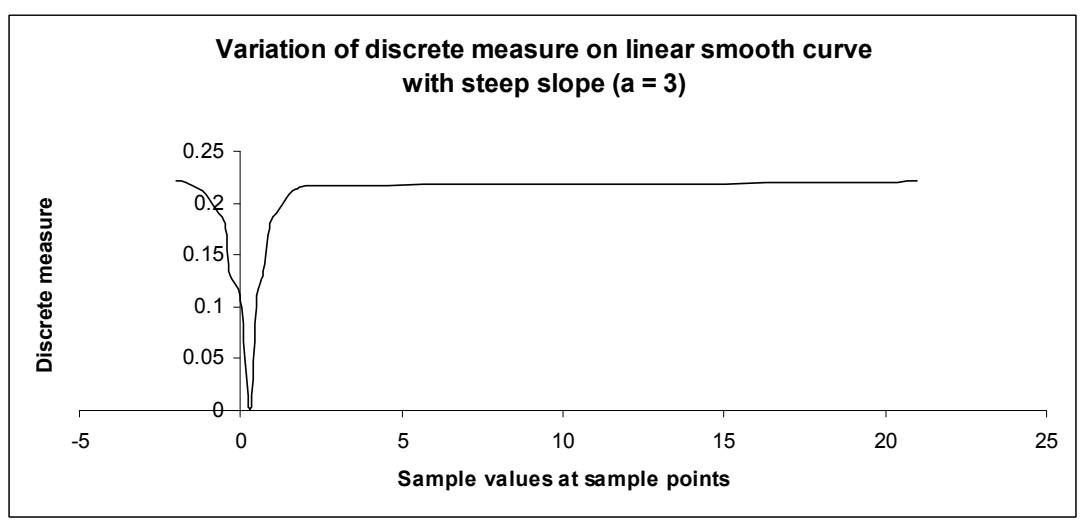

Figure 4. Variation of discrete measure on linear smooth curve with slope $a=3$.

The composite discrete measures reach zero early in the slant smooth curve as compared to the curve with steep slope. Moreover, the measure tends to saturate at higher positive sample points. In both cases, the characteristics of measure are non-linear and approximately symmetric with respect to zero measure. 


\subsection{Evaluation on Non-Linear Curves}

The evaluations of composite discrete measure on non-linear curves are carried out based on periodic and non-periodic (arbitrary) smooth functions. The generalized form of composite discrete measure is formulated in uncorrelated $A$ as given below.

$$
p\left(y_{j}, e_{j}\right)=\left\{\begin{array}{l}
e_{j} y_{j},\left(y_{j}<1\right) \wedge\left(e_{j}<1\right) \\
e_{j} / y_{j},\left(y_{j} \geq 1\right) \wedge\left(e_{j}<y_{j}\right) \wedge\left(e_{j} y_{j}>0\right) \\
y_{j} / e_{j},\left(y_{j} \geq 1\right) \wedge\left(e_{j}>y_{j}\right) \wedge\left(e_{j} y_{j}>0\right)
\end{array}\right.
$$

Initially, a trigonometric periodic smooth curve (sinusoid) is considered for evaluating the measure. The profile of the periodic smooth curve is illustrated in Figure 5.

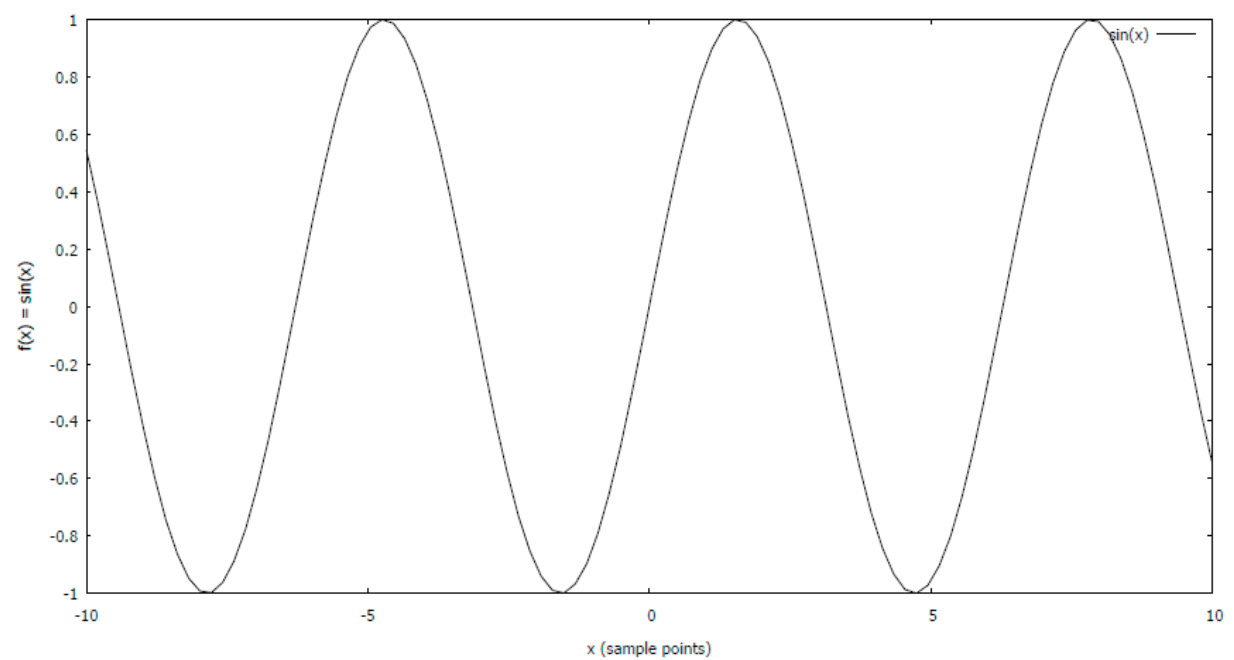

Figure 5. Sinusoid for discrete measure on periodic smooth curve.

The characteristic of the composite discrete measure on the periodic smooth curve is presented in Figure 6. In this case, the measure values decreased sharply within a narrow domain and reach zero synchronously with the periodic function. The measure exhibits a monotonic decrease in the positive domain at an extremely low rate. The profile of the measure is asymmetric in nature with respect to zero.

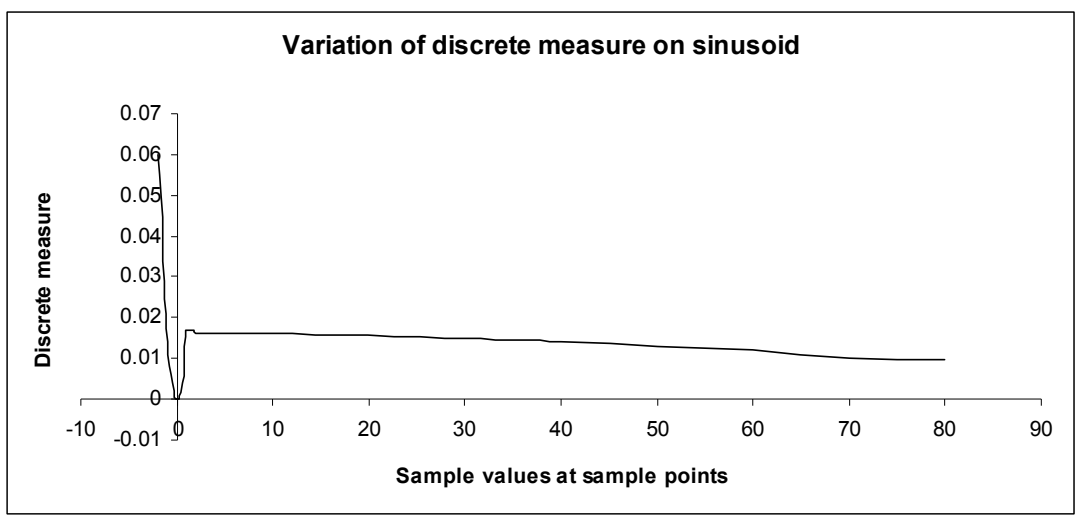

Figure 6. Variation of discrete measure at sample points on sinusoid.

Next, the composite discrete measures are computed on arbitrary smooth function and the profile of the function is illustrated in Figure 7. The curve is generated by software by using a randomized seeded function. 


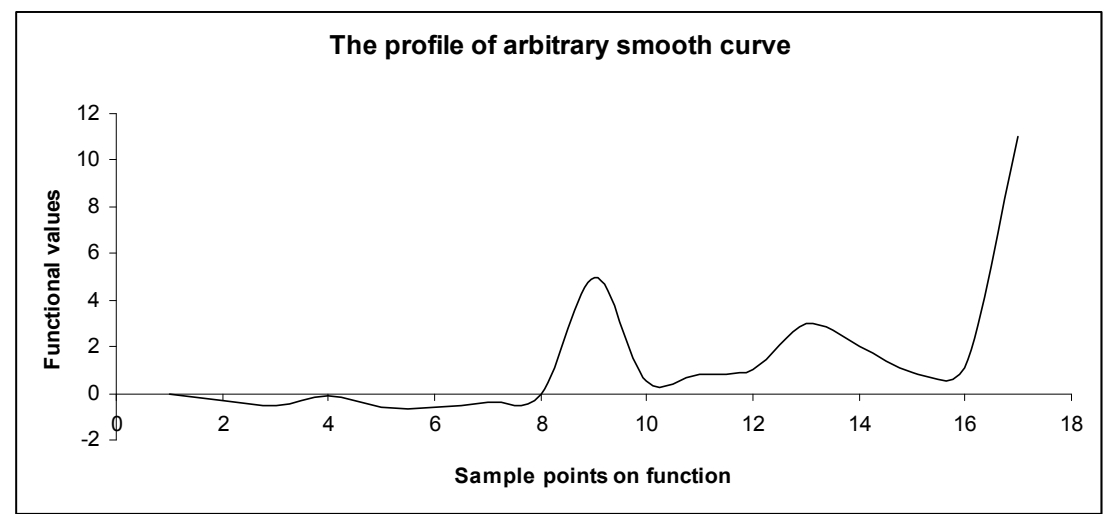

Figure 7. Profile of arbitrary smooth curve $f(x)$.

The characteristic variation in composite discrete measure on the arbitrary smooth curve is presented in Figure 8.

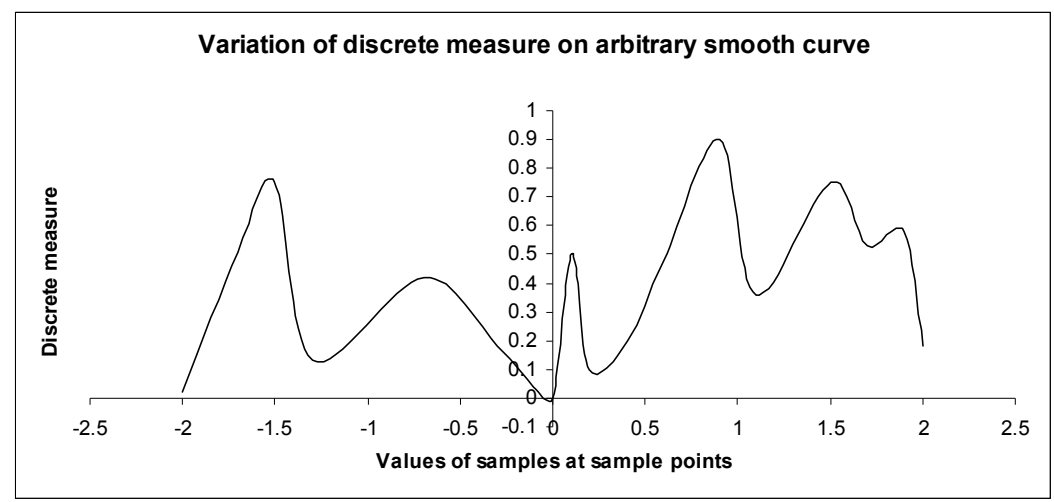

Figure 8. The variation of discrete measure on $f(x)$.

The profile of the measure illustrates that the estimated discrete measure values are random both in positive and negative domains. This indicates that the measure follows the inherent randomness of the function. Moreover, the measure is asymmetric in nature with respect to zero.

\subsection{Evaluation in z-Plane}

The evaluation of the composite discrete measure in the z-plane considers a set of uncorrelated points in complex space. The general form of a smooth and non-linear measure function is defined as,

$$
p_{c}\left(y_{j}, f_{c}\left(z_{j}\right)\right)=1 /\left(1+e^{y_{j} z_{j} z_{j} *}\right)
$$

The profile of trajectory of a point in z-plane and the associated distribution of sample points on the curve are illustrated in Figures 9 and 10, respectively. 


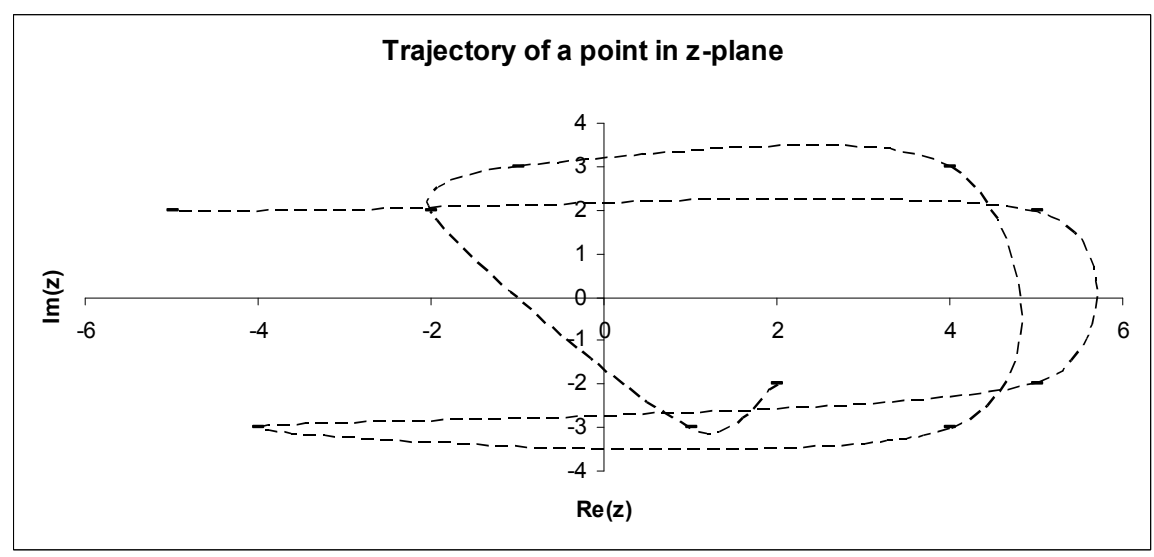

Figure 9. Curvilinear trajectory of a point in z-plane.

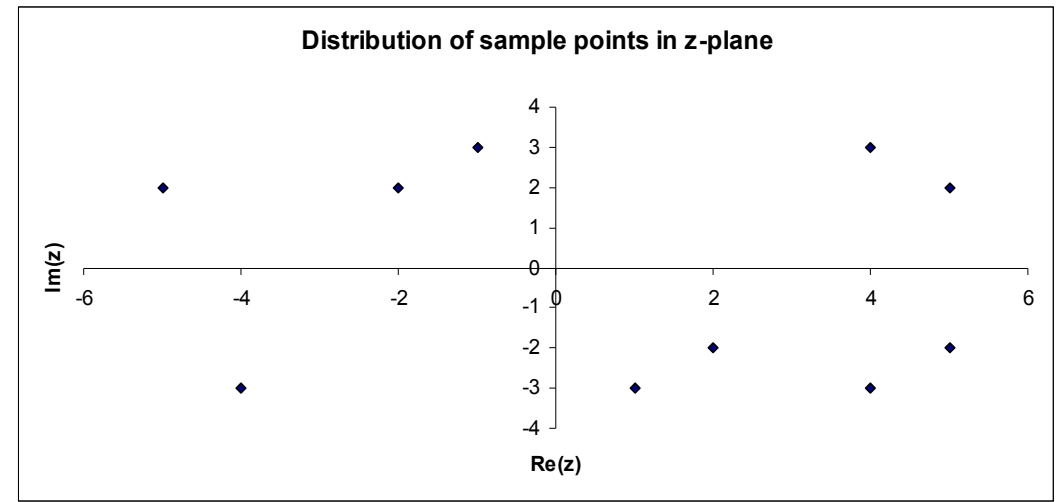

Figure 10. Distribution of sample points in z-plane on curve.

The dynamics of composite discrete measure in complex metric space on the curve is illustrated as the surface map illustrates in Figure 11.

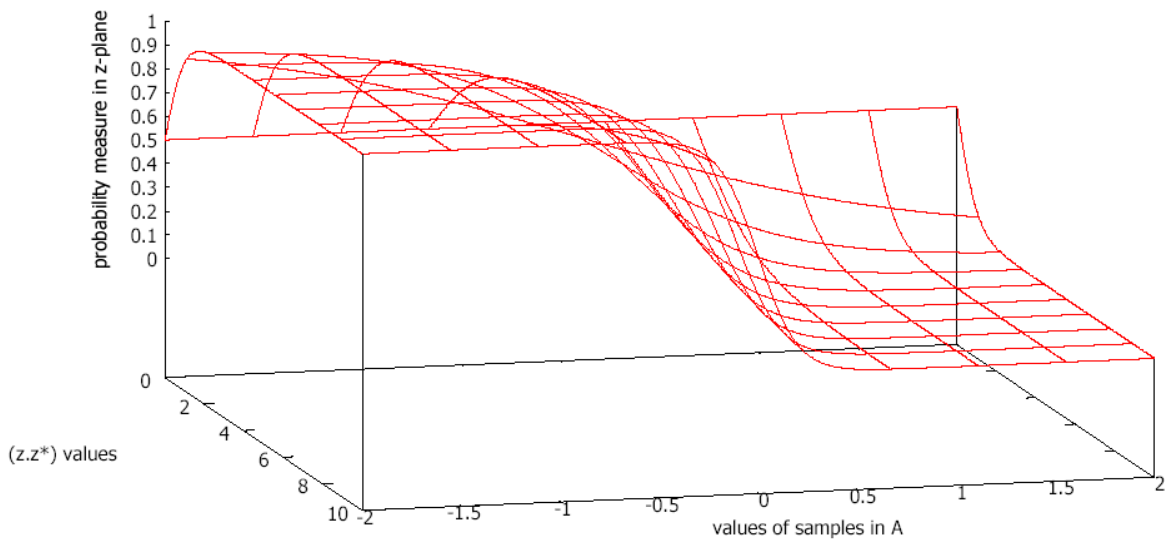

Figure 11. Surface map of discrete measure in z-plane.

The surface map illustrates that the composite discrete measure in the z-plane is highly non-linear in nature and there is a zone of sharp reduction starting from the left-neighborhood of zero in the set of samples. At the pairs of points in complex space on the extreme left of zero-th sample on the corresponding axis, the dynamics of the measure are non-linear with initial exponential increment to maxima. Moreover, as the complex conjugate values are increased within a region of sample space, the composite discrete measure decreases rapidly. 


\subsection{Measure on Real Surface}

The computation of discrete measure on real surface is carried out with the following definition of decomposed surface function,

$$
\begin{aligned}
& x \in I \subset R, y \in J \subset R, \\
& u(x)=(x+1)^{2}, \\
& v(y)=e^{\sin y}
\end{aligned}
$$

The first component of the surface function is a non-linear monotonic increase in nature, while the second component of the surface function is providing periodic breaking on the growth of the surface. The profile of the surface map is illustrated in Figure 12.

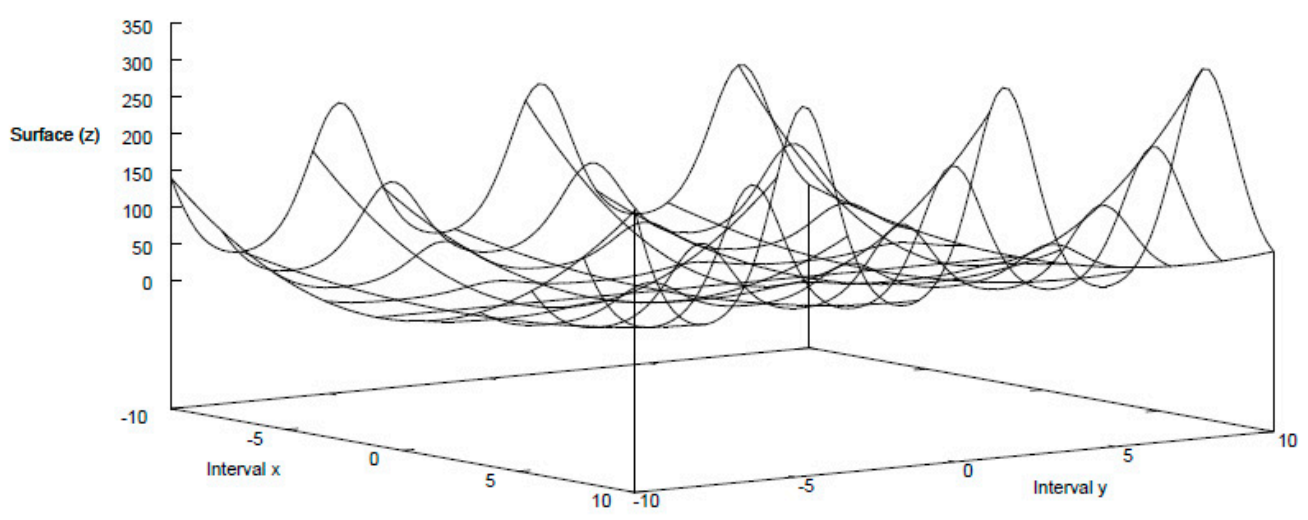

Figure 12. Profile of non-linear surface map function.

The compositely measurable function $g($.$) on the surface is considered as constant (=1)$ in order to detect the characteristics of discrete measure on surface in the absence of any external influence. The disjoint measure intervals are defined as,

$$
\begin{aligned}
& 0<m<\sup (f(\beta)), M \in(\sup (f(\beta)),+\infty) \\
& S=\{m, M\} \\
& \gamma \in S: p_{x y}=\left|\frac{1}{\gamma-f(\beta)}\right|
\end{aligned}
$$

The discrete measure on surface is computed in two cases, Case I: boundary is kept lower, and Case II: boundary is extended to a larger value. In Case I, the boundary is set to 50 and in Case II the boundary is extended seven times to 350 .

The variations of discrete measures on the surface for $m=50$ and $M=350$ are illustrated in Figures 13 and 14, respectively.

Evidently, the measures are non-linear, continuous and aperiodic in nature in both the cases. However, if the measure threshold is kept embedded into the surface, then the discrete measure never reaches zero throughout the interval and for the negative samples in the surface, the discrete measures produce enhanced positive concentration at distinct zones. On the contrary, if the measure threshold is shifted away from the surface (i.e., above the surface), then the enhanced positive concentration of discrete measure is produced only at a positive extreme. However, in both the cases, the discrete measures never reach zero. The corresponding profile of composite distribution of discrete measure surface is illustrated in Figure 15. 


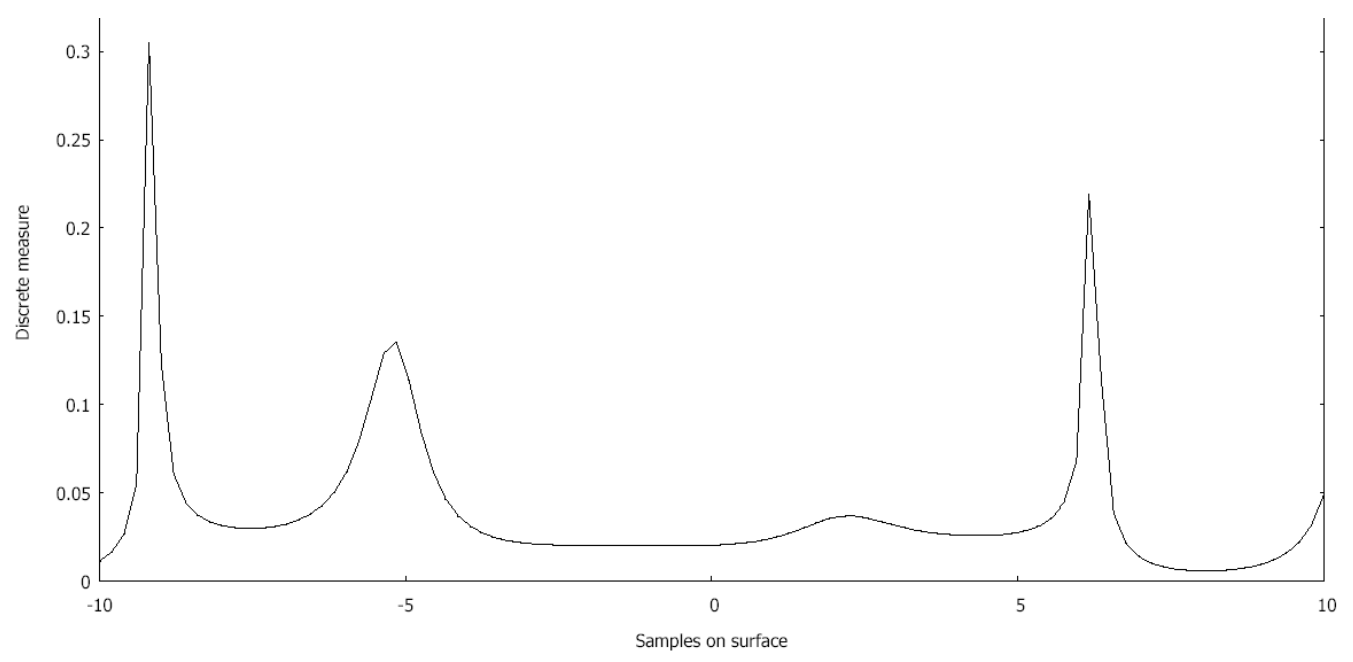

Figure 13. Discrete measure of constant function $g($.$) on surface (m=50)$.

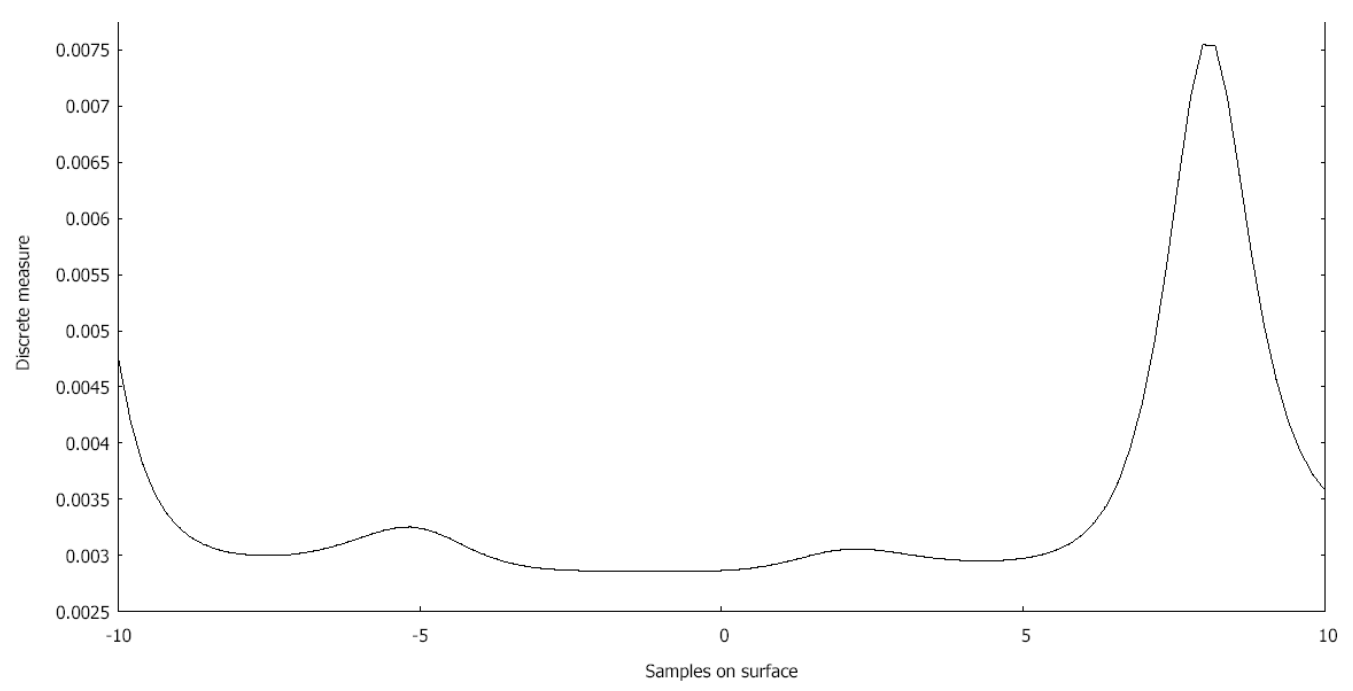

Figure 14. Discrete measure of constant function $g($.$) on surface (M=350)$.

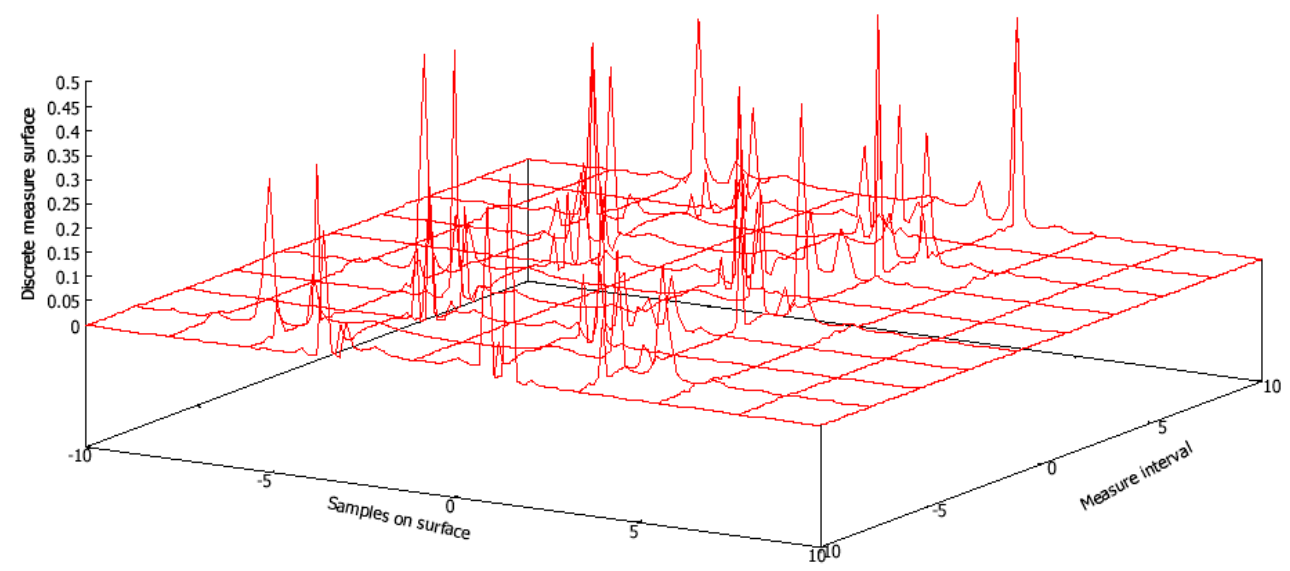

Figure 15. Distribution of discrete measure surface.

The surface map illustrates that there exists a set of distinct concentration zones and the concentration zones are distributed unevenly on the surface. Furthermore, the concentration zones are distributed throughout the measure interval. The distributions of samples do not stretch the concentration zones perpendicularly with respect to measure interval. 


\section{Comparative Analysis}

The measure theoretic analysis is applicable in Lebesgue measurable (LM) spaces and in probabilistic measures on group (PMG) structures [31]. On the other hand, the discrete composite measures (DCM) consider arbitrary functions while constructing probability measures in composite form. Furthermore, DCM considers real as well as complex metric spaces while formulating composite measures. The comparative analysis of related models is presented in Table 1.

Table 1. Comparative analysis of models of measures.

\begin{tabular}{ccccc}
\hline Models & $\begin{array}{c}\text { Locally } \\
\text { Compactness }\end{array}$ & $\begin{array}{c}\text { Haar Measurability } \\
\text { Condition }\end{array}$ & Norm Closure & Amenability \\
\hline PMG & Yes & Yes & Finite & Integrable (convergent to 0) \\
\hline DCM & Yes & No & Finite & Summable (convergent in positive interval) \\
\hline LM & No & No & Possibly infinite & Integrable (may not be convergent) \\
\hline
\end{tabular}

In general, the PMG assumes a non-commutativity property of the groups. Moreover, the space in PMG is assumed to be locally compact. The LM does not require restriction of local compactness because Lebesgue measure is equipped to operate on open intervals. However, the proposed DCM model in this paper considers that underlying space is locally compact to generate a finite probability measure. The similarity between LM and DCM is that both are not dependent on the Haar measurability condition. On the contrary, the PMG model requires the Haar measurability condition to be maintained in groups. The norm closure for PMG and DCM is finite in probability spaces, whereas the LM does not require the measure to be strictly finite. In terms of the amenability property, PMG and LM are continuous measures. However, amenability in PMG is convergent to zero, whereas in LM, such convergence may not always be guaranteed. On the other hand, the amenability property of DCM is based on summability (discrete in nature) and is convergent in a closed finite interval.

\section{Conclusions}

The computational applications such as randomized and probabilistic distributed algorithms and probabilistic databases benefit from drawing the inputs from various composite probability measures. The variability of input to probabilistic systems becomes restrictive in standard probability spaces, having long periodicity. The formulation of composite measures in different metric spaces enhances the variability of inputs. The characteristics of composite discrete measures on different metric spaces have distinguishing effects on respective computational output profiles. The computable measures can be formed on linear, periodic or arbitrary smooth curves in different metric spaces comprised of real and complex spaces. Furthermore, the corresponding algebra and monotone class property of the measures in spaces are identified and analyzed. The composite measures on 2-D real surface and condensation measures of uniform contraction map are formulated. A set of composite measures are computationally evaluated in real as well as complex space. The corresponding surface maps provide dynamics of the composite measures. The future work will include evaluations of the behaviours of probabilistic computational applications under the influence of randomized inputs drawn from the proposed composite models.

Funding: The funding of this work covered by BK21+, Gyeongsang National University, ROK.

Acknowledgments: The author would like to thank the editors and anonymous reviewers for their helpful comments and suggestions.

Conflicts of Interest: The author declares no conflict of interest. 


\section{References}

1. Hassin, Y.; Peleg, D. Distributed Probabilistic Polling and Applications to Proportionate Agreement. In Lecture Notes in Computer Science; Wiedermann, J., van Emde Boas, P., Nielsen, M., Eds.; Springer: Berlin, Germany, 1999; Volume 1644, pp. 402-411.

2. Benjelloun, O; Sarma, A.; Halevy, A.; Widom, J. ULDBs: Databases with uncertainty and lineage. In Proceedings of the 32nd International Conference on Very Large Data Bases, Seoul, Korea, 12-15 September 2006; pp. 953-964.

3. Jampani, R.; Xu, F.; Wu, M.; Perez, L.; Jermaine, C. MCDB: A monte carlo approach to managing uncertain data. In Proceedings of the 2008 ACM SIGMOD International Conference on Management of Data, New York, NY, USA, 9-12 June 2008.

4. Kent, P.; Jensen, R.K.; Kongsted, A. A comparison of three clustering methods for finding subgroups in MRI, SMS or clinical data: SPSS TwoStep Cluster analysis, Latent Gold and SNOB. BMC Med. Res. Methodol. 2014, 14, 113. [CrossRef] [PubMed]

5. Norman, G. Analyzing Randomized Distributed Algorithms. In Validation of Stochastic Systems; Baier, C., Haverkort, B.R., Hermanns, H., Katoen, J.P., Siegle, M., Eds.; Springer: Berlin, Germany; Volume 2925, pp. 384-418.

6. Wigderson, A. Do probabilistic algorithms outperform deterministic ones? In Lecture Notes in Computer Science; Larsen, K.G., Skyum, S., Winskel, G., Eds.; Springer: Berlin, Germany, 1998; Volume 1443, pp. $212-214$.

7. Calafiore, G.C.; Dabbene, F.; Tempo, R. Randomized algorithms for probabilistic robustness with real and complex structured uncertainty. IEEE Trans. Autom. Control 2000, 45, 2218-2235. [CrossRef]

8. Chung, K.M.; Pettie, S.; Su, H.H. Distributed Algorithms for the Lovasz Local Lemma and Graph Colouring. In Proceedings of the ACM Symposium on Principles of Distributed Computing, Paris, France, 15-18 July 2014.

9. Jibrin, S.; Boneh, A.; Caron, R.J. Probabilistic algorithms for extreme point identification. J. Interdiscip. Math. 2007, 10, 131-142. [CrossRef]

10. Richard, M.K. An Introduction to Randomized Algorithms. Discret. Appl. Math. 1991, 34, 165-201.

11. Dubhashi, D.; Grable, D.A.; Panconesi, A. Near-optimal, distributed edge colouring via the nibble method. Theor. Comput. Sci. 1998, 203, 225-251. [CrossRef]

12. Barenboim, L.; Elkin, M.; Pettie, S.; Schneider, J. The Locality of Distributed Symmetry Breaking. J. ACM 2016, 63. [CrossRef]

13. Lassaigne, R.; Peyronnet, S. Probabilistic verification and approximation. Ann. Pure Appl. Logic 2008, 152, 122-131. [CrossRef]

14. Repovš, D.; Savchenko, A.; Zarichnyi, M. Fuzzy Prokhorov metric on the set of probability measures. Fuzzy Sets Syst. 2011, 175, 96-104. [CrossRef]

15. Hoyrup, M.; Rojas, C. Computability of probability measures and Martin-Löf randomness over metric spaces. Inf. Comput. 2009, 207, 830-847. [CrossRef]

16. Tian, Y.; Yin, Z.; Huang, M. Missing data probability estimation-based Bayesian outlier detection for plant-wide processes with multisampling rates. Symmetry 2018, 10, 475. [CrossRef]

17. Shao, S.; Zhang, X.; Li, Y.; Bo, C. Probabilistic single-valued (interval) neutrosophic hesitant fuzzy set and its application in multi-attribute decision making. Symmetry 2018, 10, 419. [CrossRef]

18. Ercan, S. On the statistical convergence of order $\alpha$ in paranormed space. Symmetry 2018, 10, 483. [CrossRef]

19. Breugel, F.; Worrell, J. A behavioural pseudometric for probabilistic transition systems. Theor. Comput. Sci. 2005, 331, 115-142. [CrossRef]

20. Kurtz, T.G.; Manber, U. A probabilistic distributed algorithm for set intersection and its analysis. Theor. Comput. Sci. 1987, 49, 267-282. [CrossRef]

21. Hind, I.; Ali, D. Probabilistic distributed algorithm for uniform election in polyo-triangular grid graphs. In Proceedings of the 2014 Second World Conference on Complex Systems (WCCS), Agadir, Morocco, 10-12 November 2014.

22. Anashin, V.; Khrennikov, A. Applied Algebraic Dynamics; Walter de Gruyter: Berlin, Germany, 2009.

23. Eifler, L. Open mapping theorems for probability measures on metric spaces. Pac. J. Math. 1976, 66, 89-97. [CrossRef] 
24. Parthasarathy, K.R. Probability measures in a metric space. In Probability and Mathematical Statistics; Academic Press: New York, NY, USA, 1967; pp. 26-55.

25. Horvath, A.G. Normally Distributed Probability Measure on the Metric Space of Norms. Acta Math. Sci. 2013, 33, 1231-1242. [CrossRef]

26. Vovk, V.; Shen, A. Prequential randomness and probability. Theor. Comput. Sci. 2010, 411, $2632-2646$. [CrossRef]

27. Hertling, P.; Weihrauch, K. Randomness spaces. In Lecture Notes in Computer Science; Larsen, K.G., Skyum, S., Winskel, G., Eds.; Springer: Berlin, Germany, 1998; Volume 1443, pp. 796-807.

28. Edalat, A. The Scott topology induces the weak topology. In Proceedings of the 11th Annual IEEE Symposium on Logic in Computer Science, Washington, DC, USA, 27-30 July 1996.

29. Gács, P. Uniform test of algorithmic randomness over a general space. Theor. Comput. Sci. 2005, 341, 91-137. [CrossRef]

30. Calude, C.S.; Hertling, P.H.; Jurgensen, H.; Weihrauch, K. Randomness on full shift spaces. Chaos Solitons Fractals 2001, 12, 491-503. [CrossRef]

31. Willis, G.A. Probability measures on groups and some related ideals in group algebras. J. Funct. Anal. 1990, 92, 202-263. [CrossRef]

(C) 2018 by the author. Licensee MDPI, Basel, Switzerland. This article is an open access article distributed under the terms and conditions of the Creative Commons Attribution (CC BY) license (http:/ / creativecommons.org/licenses/by/4.0/). 